\title{
Anaemia and incidence of post stroke dementia
}

Running Head: Anaemia and post-stroke dementia

Phyo Kyaw Myint ${ }^{1}$, Mary Joan Macleod ${ }^{2}$, Allan B Clark ${ }^{3}$, Toby O Smith ${ }^{4}$, Joao H.

Bettencourt-Silva ${ }^{5}$, Anthony Kneale Metcalf ${ }^{3,5}$, John F. Potter ${ }^{3,5}$

${ }^{1}$ Institute of Applied Health Sciences, University of Aberdeen, UK

${ }^{2}$ Institute of Medical Sciences, University of Aberdeen, UK

${ }^{3}$ Norwich Medical School, University of East Anglia, UK

${ }^{4}$ Nuffield Department of Orthopaedics, Rheumatology and Musculoskeletal Sciences, University of Oxford, UK

${ }^{5}$ Norfolk and Norwich University Hospital, Norwich, UK

Phyo K. Myint, Professor of Medicine of Old Age

Mary Joan Macleod, Senior Lecturer, Medicine \& Therapeutics

Allan B. Clark, Senior Lecturer in Medical Statistics

Toby O. Smith, Senior Researcher in Rehabilitation

Joao H. Bettencourt-Silva, Research Associate

Anthony Kneale Metcalf, Consultant Stroke Physician

John F. Potter, Professor of Ageing \& Stroke Medicine

Correspondence to:

Professor Phyo Kyaw Myint,

Room 4:013 Polwarth Building,

School of Medicine, Medical Sciences \& Nutrition

University of Aberdeen, Foresterhill,

Aberdeen, AB25 2ZD, UK.

Tel: +44 (0) 1224437841 ,

Fax: +44 (0) 1224437911 ,

Email: phyo.myint@abdn.ac.uk

Conflict of Interest: NONE 


\section{Abstract}

Objectives: To assess the impact of anaemia on incidence of post-stroke dementia.

Patients and Methods: We used data from a UK regional stroke register. To be eligible, patient must have survived to discharge and had anaemia by WHO criteria. Dementia status and other prevalent co-morbidities were assessed using ICD-10 codes. Patients were followed till May 2015 (mean follow-up 3.7 years, total person years = 27,769). Hazard Ratio for incident dementia was calculated using Cox-proportional hazards model controlling for potential confounders. Fine and Gray model was additionally constructed using mortality as the competing risk.

Results: A total of 7,454 stroke patients were included with mean age (SD) of 75.9(12.3) years $(50.2 \%$ men). Those with anaemia were older, has higher disability and co-morbidity burden prior to stroke. We observed a large amount of variation in the dementia incidence rates over time and that the hazard ratio increased every year. The significant association between anaemia and dementia incidence was lost after controlling for pre-stroke Modified Rankin score (HR1.17(0.97,1.40)). With every $20 \mathrm{~g} / \mathrm{dL}$ increase in $\mathrm{Hb}$ was associated with a significant reduction in the risk of dementia after adjustment for age, sex, stroke factors and disability but lost significance after adjustment for vascular risk factors. Competing risk analyses showed similar results.

Conclusion: Whilst we found no evidence of anaemia as a risk factor for post-stroke dementia, the findings may be limited by potential under recognition of post stroke dementia. 


\section{Introduction}

Anaemia is common in patients with acute stroke. The prevalence figure of up to $30 \%$ has been reported in hospital-based studies [1][2]. Our recent work demonstrated that $25 \%$ of stroke patients were anaemic at the time of hospital admission [3]. The aetiology of dementia after a stroke or transient ischaemic attack (TIA) has been highlighted in research [4]. The link between anaemia and cognition has also grown a considerable interest over the last few decades [5][6][7]. Systematic reviews and meta-analyses published in this area suggest potential link between anaemia and cognitive decline or dementia in older aged cohorts [8][9][10].

With post-stroke dementia being a significant issue, there is now a pressing need for greater understanding of risk factors for dementia in this patient group. One potential risk factor may be anaemia as suggested in general populations [9][10]. The proposed pathophysiology mechanisms include direct effect of chronic cerebral hypoxia., as a marker of diseases and processes such as ischemic and lower erythropoietin levels increasing the risk of neuronal degeneration. Others include iron dysregulation associated with increased brain oxidative stress, and chronic cerebral hypoperfusion in the prefrontal cortex or inflammatory neurodegenerative processes. It was also suggested that anaemia may cause the progression of white matter disease through low physical function with heightened vascular disease risk [9]. Despite different pathological mechanisms, post stroke dementia occurs as the result of vascular dementia or mixed (Alzheimer's and vascular dementia) and thus regarded as a single clinical entity. 
However, few studies have examined this link in stroke patients. Given that stroke has high mortality in the first year and the fact that anaemia contribute this mortality risk [3], it is unclear whether stroke survivors who were anaemic would have either increased or decreased risk of post-stroke dementia.

\section{Patients and Methods}

The study population consisted of 7,454 stroke patients consecutively admitted between January 2003 - May 2015 to Norfolk and Norwich University Hospital, a regional tertiary centre in East Anglia, UK, with a catchment population of approximately 750,000. Ethical approval was obtained from the Newcastle and Tyneside National Health Service (NHS) Research Ethics Committee (12/NE/0170) and the study protocol was approved by the Steering Committee of the Register. Of 11,727 entries, 991 repeated entries (with second or third stroke recorded in the register were excluded. After further exclusion of 2,404 who died as in-patient, there were a total of 8,332 who were discharged from hospital for the first recorded stroke in the register, 379 had previous history of dementia prior to stroke and therefore excluded. 499 participants did not have haemoglobin values and had missing data for other co-variates and thus further excluded.

The data collection methods for this prospective hospital-based register have been previously reported [11]. Briefly, the data were obtained from paper and electronic records, reviewed and then entered onto the register database by the hospital stroke data team and vetted by clinical team members for accuracy. For each patient admitted, the pre-stroke modified Rankin score (mRS), as modified by UK-TIA investigators [12], was ascertained from nursing and medical records by stroke specialist nurses. At discharge, the dead or alive status 
was recorded to capture in-hospital mortality. Follow-up for mortality was obtained by electronic record linkage with Office of National Statistics data through hospital episodes in May 2015.

To be eligible in the current study, patients could not have history of dementia (any type) prior to stroke and they must have survived to hospital discharge. Haemoglobin level obtained closed to discharge was used to determine the $\mathrm{Hb}$ or anaemia status. Only confirmed cases of stroke were included. Stroke was diagnosed using evidence from clinical features and neuroimaging (typically CT and in some cases MRI). Anaemia was defined according to the WHO criteria of $\mathrm{Hb}<12.0 \mathrm{~g} / \mathrm{dL}$ in females and $<13.0 \mathrm{~g} / \mathrm{dL}$ in males and elevated haemoglobin was defined as $>15.5 \mathrm{~g} / \mathrm{dL}$ in females and $>17.0 \mathrm{~g} / \mathrm{dL}$ in males [13].

The variables included were age, sex, stroke sub-type (ischaemic/haemorrhagic), pre-stroke disability depicted by modified Rankin score (mRS), Oxfordshire Community Stroke Project (OCSP) classification (Total Anterior Circulation Stroke, Partial Anterior Circulation Stroke, Posterior Circulation Stroke, Lacunar Stroke), haemoglobin levels at discharge, comorbidities (coronary heart disease including myocardial infarction, congestive heart failure (CHF), atrial fibrillation, hypertension, hyperlipidaemia, previous Stroke, diabetes mellitus, peripheral vascular disease, chronic obstructive pulmonary disease (COPD), chronic kidney disease (CKD), and malignancy).

Dementia incidence was captured through UK NHS record linkage system via the patient administrative system (PAS) which records both secondary care and primary care based diagnosis of dementia in the NHS setting. International Classification of Disease (ICD) 10 
codes F00, F01, F02 and F051 were used to capture both prevalent and incident dementia. Statistical analysis

Characteristics of the individuals with and without anaemia were summarized using descriptive statistics and compared using a chi-squared test for categorical variables and a ttest for continuous variables. Annual incidence rates of stroke were calculated for each calendar year of follow-up stratified by year of study entry, excluding individuals who died in the year from the denominator.

The association between anaemia and dementia incidence was described using Kaplan-Meier plots and estimated using a cox proportional hazards model. This was done for six separate models A-F using hierarchical adjustments. The association was adjusted for age and sex in model A; model B adjusted additionally for stroke characteristics, model $\mathrm{C}$ additionally adjusted for pre-stroke modified Rankin score, model D additionally adjusted for key risk factors for dementia namely atrial fibrillation, coronary heart disease, previous stroke or TIA, peripheral vascular disease and history of hypertension. Model E additionally adjusted for other major co-morbidities which included COPD, CKD, CHF, hyperlipidaemia, diabetes, and malignancy. Model $\mathrm{F}$ additionally adjusted for year of stroke to account for the possibility that dementia diagnoses changed of the course of the follow-up period. The association between haemoglobin $(\mathrm{Hb})$ and stroke incidence was assessed in a similar fashion using the same models. No major departures from the model assumptions were observed. As anaemia is also associated with death a competing risk model (Fine and Gray) was fitted using the same modelling strategies as above. 


\section{Results}

A total of 7,454 stroke patients were included in the current report; their mean age (SD) was $75.9(12.3)$ years and there were $3,742(50.2 \%)$ men. (mean follow up $=3.7$ years, median follow up $=2.9$ years, total person years $=27,769$ years). Table 1 shows the sample characteristic comparison between those with and without anaemia at the time of discharge by sex specific analysis. It demonstrates that most factors considered are significantly different between individuals with anaemia and those without; those with anaemia tended to be older and significantly more likely to be more disabled prior to stroke with higher burden of co-morbidities.

Figure 1 shows the Kaplan Meier Curve showing linear trends in development of dementia in patients without (top line) and with (bottom line) anaemia. Supplementary Table 1 shows that there is a large amount of variation in the incidence rates over time. Kaplan-Meier plot with each year being a separate line demonstrate clearly that the hazard ratio has generally increased every year (apart from 2015 which does not have full year of follow-up) (see Figure 2).

Table 2 shows the estimated hazards ratios and corresponding 95\% confidence intervals. This shows a significant association between anaemia and dementia incidence, but this loses significance on adjusted for pre-stroke mRS. A similar pattern is observed when $\mathrm{Hb}$ was entered into model as a linear variable (with every $20 \mathrm{~g} / \mathrm{dL}$ increase) with a significant reduction in the risk of dementia until adjustment for key risk factors of dementia. Once competing risks are accounted for, this association loses significant in all but model A. This is suggestive that the association observed before adjustment is due to the imbalance in the 
characteristics at baseline between the two groups and that once the competing risk of death is taken into account the association is not significant.

\section{Discussion}

To the best of our knowledge, we are the first group to report the association between presence or absence of anaemia or haemoglobin levels and incidence of dementia over longterm follow-up in a large cohort of unselected stroke survivors. Our study results highlight the importance of death as the competing risk in development of post-stroke dementia, which carries substantial mortality risk after hospital discharge. Whilst we did not demonstrate the clear dose-response relationship between anaemia and dementia risk, Kaplan-Meier curves demonstrate the potential effect of whether diagnosis of dementia was made after stroke. Given that dementia diagnosis is based on progressive nature of clinical assessment criteria, this complex nature should be taken into account in risk prediction studies of post-stroke dementia.

The key strengths of the study include relatively large sample of unselected consecutive stroke admissions. We were also able to control for acute stroke factors and comprehensive list of co-morbidities as well as functional disability. We were able to follow up for longterm with minimal loss to follow-up due to NHS record linkage. In UK, almost everyone has a NHS number and Office of National Statistics data are linked to local NHS databases and vital status including mortality are ascertained regularly. Local NHS record linkage allows us to determine the date of dementia diagnosis appearing in health records and therefore we 
were able to estimate hazards ratios rather than odds ratio. We were able to robustly considered death as competing risk by Fine \& Gray methods [14].

Indeed, numerous risk factors for cognitive decline and dementia have been previously reported in stroke survivors. These included age, history of cardio-metabolic disease, atrial fibrillation, lower educational attainment, stroke severity, aphasia, recurrent stroke, pre-stroke cognitive impairment, and neuroimaging makers such as medial temporal lobe atrophy and hippocampal changes [15]. Currently developed are complex [16][17][18] and it is unlikely to be of clinical utility. For prediction tools to be of clinical utility, criteria should include readily available parameters that can be reliably measured with minimal resources. Discharge haemoglobin level offers a potential role given that there are several plausible pathophysiological mechanistic links between anaemia and post-stroke dementia. Anaemia could lead to chronic reduction of cerebral oxygenation secondary to decreased oxygencarrying capacity of the blood. Alternatively, it serves as a marker of diseases and processes such as ischemic and lower erythropoietin levels, thereby increasing the risk of neuronal degeneration, iron dysregulation and associated with increased brain oxidative stress [19]. Anaemia could also be associated with the progression of white matter disease [20]. Another potential causal pathway is that it could operate through reduced physical function due to tiredness secondary to anaemia. It was proposed that cardiorespiratory fitness is linked to executive functions through an increase in brain derived neurotrophin factor or a parasympathetic related increase in efficiency of prefrontal neural function [21]. Therefore, presence of functional impairment as the results of stroke damage may increase the risk of dementia in anaemic older people. 
We observe that the significant relationship between anaemia and dementia risk was attenuated after controlling for pre-stroke disability assessed using modified Rankin scale and other risk factors. This is in agreement with results from an early systematic review on this topic [22]. We observe the higher risk of dementia associated with the latter years of the study suggesting there may be bias towards under diagnosis of dementia in earlier years of this study. Of note, dementia diagnosis has changed over the study period; changing trends in diagnosis of dementia in the UK has been reported between 2005 and 2015. The proportion of people diagnosed with dementia in the UK doubled from $0 \cdot 42 \%(19,635$ of 4,640,290 participants) in 2005 to $0 \cdot 82 \%$ (25,925 of 3,159,754 participants) in 2015 ( $\chi^{2}$ test for trend, $\mathrm{p}<0 \cdot 0001)[23]$.

There are some limitations. As a registry based observational study we were not able to fully adjust for treatment effects (e.g. blood transfusion, use of iron supplements and erythropoietin stimulating agents) after hospital discharge which might have attenuated the actual effect size of the relationship between anaemia of low $\mathrm{Hb}$ levels and subsequent risk of dementia. We were unable to take into account the duration of anaemia or assess the impact of abnormal haemoglobin levels after hospital discharge. We did not stratify analysis by anaemia subtype as this will reduce the number of outcomes (dementia) further unlikely to produce any meaningful results due to loss of power. We also did not control for some haematological parameters and biological parameters such as B12, folate levels and kidney function which may have confounded the association. As an observational study there may be residual confounding as well as effect of known or unknown confounders which were not taken into account. As a record linkage study, we were not able to confidently differentiate between different types of common dementias, although majority of cases are most likely to be either vascular dementia or dementia of mixed pathology. We also relied on dementia 
diagnosis made by clinicians in various setting evident by variation in annual diagnosis rates but perhaps this reflects the real world nature of the study. This variation may vary with time due to increasing awareness and better diagnosis. Due to relatively small sample size coupled with potential under-estimation of dementia diagnosis we were not able to examine the specific cause of anaemia and risk of dementia. It is not clear how much of cognitive decline after stroke is related to focal damage due to stroke per se, and how much is indeed contributed by other risk factors including anaemia. However, the fact anaemia may contribute cognitive decline through stroke (e.g. stroke size due to inadequate collateral circulation efficiency) makes it almost implausible to address this issue in human studies. The only way perhaps to study this would be inducing anaemia in animal models where a comparator group with same stroke type, size, and location would not have anaemia.

In summary, while we did not find strong association between anaemia and incident dementia in stroke survivors, the study should be replicated in the future with follow-up data specifically collected to diagnose dementia in stroke survivors. Future studies should also examine the link between specific type of anaemia and dementia risk in patients with stroke. With improvement in awareness of dementia after stroke and improvement in diagnosis of dementia, it presents an opportunity to revisit the actual risk estimates of previously identified risk factors as well as potential new risk factors with the view of developing simple prediction rule that is pragmatic and applicable in low resource setting thus useful for daily clinical practice. 


\section{Acknowledgements}

We gratefully acknowledge the stroke data team at the NNUH Foundation NHS Trust. We gratefully acknowledge Professor Bowles who is one of the steering committee members of the NNUH Stroke and TIA register.

\section{Contributors}

PKM and TOS conceived the idea. JHBS performed record linkage. ABC analysed the data. Draft manuscript was prepared by PKM and TOS and all authors made contribution to data interpretation and writing of the paper. PKM is the guarantor.

\section{Source of Funding Declarations}

Dr Toby Smith is supported by funding from the National Institute for Health Research (NIHR) Oxford Health Biomedical Research Centre. The views expressed are those of the author(s) and not necessarily those of the NIHR.

\section{Conflict of Interest}

All authors declare no conflict of interest. 


\section{References}

[1] Sico JJ, Concato J, Wells CK, Lo AC, Nadeau SE, Williams LS, Peixoto AJ, Gorman M, Boice JL, Bravata DM. Anaemia is associated with poor outcomes in patients with less severe ischaemic stroke. J Stroke Cerebrovasc Dis 2013; 22:271-278.

[2] Hao Z, Wu B, Wang D, Lin S, Tao W, Liu M. A cohort study of patients with anaemia on admission and fatality after acute ischaemic stroke. J Clin Neuroscience 2013; 20:37-42.

[3] Barlas RS, Honney K, Loke YK, McCall SJ, Bettencourt-Silva JH, Clark AB, Bowles KM, Metcalf AK, Mamas MA, Potter JF, Myint PK. Impact of Haemoglobin Levels and Anaemia on Mortality in Acute Stroke: Analysis of UK Regional Registry Data, Systematic Review, and Meta-Analysis. J Am Heart Assoc. 2016 Aug 17;5(8).

[4] Hénon H, Pasquier F, Leys D. Poststroke dementia. Cerebrovasc Dis. 2006;22(1):61-70.

[5] Taraghi Z, Akbari Kamrani AA, Foroughan M, Yazdani J, Mahdavi A, Baghernejad SK. Cognitive impairment among elderly patients with chronic heart failure and related factors. Iran J Psychiatry Behav Sci. 2016;10(2):e4500.

[6] Dlugaj M, Winkler A, Weimar C, Dürig J, Broecker-Preuss M, Dragano N, Moebus S, Jöckel KH, Erbel R, Eisele L; Heinz Nixdorf Recall Study Investigative Group. Anaemia and mild cognitive impairment in the German general population. J Alzheimers Dis.

2016;49(4):1031-42.

[7] Jonassaint CR, Varma VR, Chuang YF, Harris GC, Yasar S, Polinder-Bos H, Carlson MC. Lower haemoglobin is associated with poorer cognitive performance and smaller brain volume in older adults. J Am Geriatr Soc. 2014;62(5):972-3.

[8] Stewart MW, Traylor AC, Bratzke LC. Nutrition and cognition in older adults with heart failure: a systematic review. J Gerontol Nurs. 2015;41(11):50-9.

[9] Andro M, Le Squere P, Estivin S, Gentric A. Anaemia and cognitive performances in the elderly: a systematic review. Eur J Neurol. 2013;20(9):1234-40.

[10] Peters R, Burch L, Warner J, Beckett N, Poulter R, Bulpitt C. Haemoglobin, anaemia, dementia and cognitive decline in the elderly, a systematic review. BMC Geriatr. 2008;8:18. doi: 10.1186/1471-2318-8-18.

[11] White JR, Bettencourt-Silva JH, Potter JF, Loke YK, Myint PK. Changes in antiplatelet use prior to incident ischaemic stroke over 7 years in a UK centre and the association with stroke subtype. Age Ageing. 2013 Sep;42(5):594-8.

[12] Farrell B, Godwin J, Richards S, Warlow C. The United Kingdom transient ischaemic attack (UK-TIA) aspirin trial: final results. J Neurol, Neurosurg and Psychiatry. 1991; 54:1044-1054. 
[13] Blanc B, Finch CA, Hallberg L, Lawkowicz W, Layrisse M, Mollin DL, Rachmilewitz M, Ramalingaswami V, Sanchez-Medal L, Wintrobe MM. Nutritional Anaemias. Report of WHO Scientific Group. World Health Organ Tech Rep Ser. 1968; 405:1-40.

[14] Fine, J. and R. Gray. 1999. A proportional hazards model for the subdistribution of a competing risk. Journal of the American Statistical Association. 94: 496-509.

[15]Tang EYH, Robinson L, Stephen BCM. Risk Prediction Models for Post-Stroke Dementia. Geriatrics. 2017; 2: 19.

[16] Stephan, B.C.; Minett, T.; Muniz Terrera, G.; Matthews, F.E.; Brayne, C. Dementia prediction for people with stroke in populations: Is mild cognitive impairment a useful concept? Age Ageing 2014.

[17] Kandiah, N.; Chander, R.J.; Lin, X.; Ng, A.; Poh, Y.Y.; Cheong, C.Y.; Cenina, A.R.; Assam, P.N. Cognitive impairment after mild stroke: Development and validation of the SIGNAL2 risk score. J. Alzheimer's Dis. 2016, 49, 1169-1177.

[18] Lin, J.H.; Lin, R.T.; Tai, C.T.; Hsieh, C.L.; Hsiao, S.F.; Liu, C.K. Prediction of poststroke dementia. Neurology 2003, 61, 343-348.

[19] Droge W, Schipper HM. Oxidative stress and aberrant signalling in aging and cognitive decline. Aging Cell 2007; 6: 367-370.

[20] Inzitari M, Zakai NA, Longstreth WT, Cushman M, Newman AB. Anaemia is associated with the progression of white matter disease in older adults with high blood pressure. $\mathrm{J} \mathrm{Am}$ Geriatr Soc 2008; 56: 1867-1872.

[21] Hansen AL, Johensen BH, Sollers JJ, Stenvik K, Thayer JF. Heart rate variability and its relation to prefrontal cognitive function. The effects of training and detraining. Eur J Appl Physiol 2004; 93: 263-272.

[22] Pendlebury, S.T.; Rothwell, P.M. Prevalence, incidence, and factors associated with prestroke and post-stroke dementia: A systematic review and meta-analysis. Lancet Neurol. 2009, 8, 1006-1018.

[23] Donegan K, Fox N, Black N, Livingston G, Banerjee S, Burns A. Trends in diagnosis and treatment for people with dementia in the UK from 2005 to 2015: a longitudinal retrospective cohort study. Lancet Public Health 2017; 2: e149-56. 


\section{Figure Legends}

Figure 1: Kaplan Meier Curves showing the incidence of dementia in patients without anaemia (top line) and with anaemia (bottom line)

Figure 2: Kaplan-Meier Curve for dementia incidence for individual year 
Table 1: Sex-specific sample characteristics comparison between stroke patients who were anaemic at discharge and those without anaemia

\begin{tabular}{|c|c|c|c|}
\hline & No Anaemia & Anaemia & $\mathrm{p}$-value \\
\hline & Total $\mathrm{N}=5019$ & Total $\mathrm{N}=2435$ & \\
\hline Men & 2863 & 879 & \\
\hline Age (mean/SD) & $71.5(12.2)$ & $78.9(10.5)$ & $<0.001$ \\
\hline \multicolumn{4}{|l|}{ Stroke Type } \\
\hline Haemorrhagic & $340(11.9 \%)$ & $66(7.6 \%)$ & $<0.001$ \\
\hline Ischaemic & $2506(88.1 \%)$ & $807(92.4 \%)$ & \\
\hline \multicolumn{4}{|l|}{ OCSP } \\
\hline LACS & $726(27.4 \%)$ & $212(25.9 \%)$ & 0.004 \\
\hline PACS & $937(35.4 \%)$ & $331(40.4 \%)$ & \\
\hline POCS & $556(21.0 \%)$ & $136(16.6 \%)$ & \\
\hline TACS & $325(12.3 \%)$ & $94(11.5 \%)$ & \\
\hline Undefined & $105(4.0 \%)$ & $46(5.6 \%)$ & \\
\hline \multicolumn{4}{|l|}{ Pre-stroke mRS } \\
\hline 0 & $2201(79.8 \%)$ & $493(60.4 \%)$ & $<0.001$ \\
\hline 1 & $275(10.0 \%)$ & $124(15.2 \%)$ & \\
\hline 2 & $126(4.6 \%)$ & $78(9.6 \%)$ & \\
\hline 3 & $97(3.5 \%)$ & $85(10.4 \%)$ & \\
\hline 4 & $46(1.7 \%)$ & $26(3.2 \%)$ & \\
\hline 5 & $13(0.5 \%)$ & $10(1.2 \%)$ & \\
\hline \multicolumn{4}{|l|}{ Comorbidities } \\
\hline Previous stroke/TIA & $587(20.9 \%)$ & $237(27.9 \%)$ & \\
\hline CHD including MI & $401(14.0 \%)$ & $231(26.3 \%)$ & $<0.001$ \\
\hline PVD & $40(1.4 \%)$ & $45(5.1 \%)$ & $<0.001$ \\
\hline $\begin{array}{l}\text { COPD (incl. } \\
\text { Emphysema/bronchitis) }\end{array}$ & $102(3.6 \%)$ & $65(7.4 \%)$ & $<0.001$ \\
\hline CKD & $39(1.4 \%)$ & $72(8.2 \%)$ & $<0.001$ \\
\hline $\begin{array}{l}\text { Congestive Cardiac } \\
\text { Failure }\end{array}$ & $125(4.4 \%)$ & $100(11.4 \%)$ & $<0.001$ \\
\hline Atrial Fibrillation & $263(9.2 \%)$ & $144(16.4 \%)$ & $<0.001$ \\
\hline Hypertension & $615(21.5 \%)$ & $345(39.2 \%)$ & $<0.001$ \\
\hline Hyperlipidemia & $104(3.6 \%)$ & $69(7.9 \%)$ & $<0.001$ \\
\hline Diabetes Mellitus & $209(7.3 \%)$ & $166(18.9 \%)$ & $<0.001$ \\
\hline Cancers & $259(9.0 \%)$ & $191(21.7 \%)$ & $<0.001$ \\
\hline Women & 2156 & 1556 & \\
\hline Age (mean/SD) & $77.3(12.1)$ & $80.5(10.8)$ & $<0.001$ \\
\hline \multicolumn{4}{|l|}{ Stroke Type } \\
\hline Haemorrhagic & $262(12.2 \%)$ & $127(8.2 \%)$ & $<0.001$ \\
\hline Ischaemic & $1887(87.8 \%)$ & $1424(91.8 \%)$ & \\
\hline \multicolumn{4}{|l|}{ OCSP } \\
\hline LACS & $581(28.8 \%)$ & $379(26.0 \%)$ & 0.026 \\
\hline PACS & $762(37.7 \%)$ & $581(39.9 \%)$ & \\
\hline POCS & $346(17.1 \%)$ & $216(14.8 \%)$ & \\
\hline TACS & $267(13.2 \%)$ & $218(15.0 \%)$ & \\
\hline
\end{tabular}




\begin{tabular}{|l|c|c|c|}
\hline Undefined & $64(3.2 \%)$ & $63(4.3 \%)$ & \\
\hline Pre-stroke mRS & & & \\
\hline 0 & $1469(70.8 \%)$ & $795(54.6 \%)$ & $<0.001$ \\
\hline 1 & $219(10.6 \%)$ & $231(15.9 \%)$ & \\
\hline 2 & $158(7.6 \%)$ & $149(10.2 \%)$ & \\
\hline 3 & $153(7.4 \%)$ & $184(12.6 \%)$ & \\
\hline 4 & $61(2.9 \%)$ & $77(5.3 \%)$ & \\
\hline 5 & $14(0.7 \%)$ & $19(1.3 \%)$ & \\
\hline Comorbidities & & & \\
\hline Previous stroke/TIA & $465(21.9 \%)$ & $408(26.9 \%)$ & $<0.001$ \\
\hline CHD including MI & $236(10.9 \%)$ & $267(17.2 \%)$ & $<0.001$ \\
\hline PVD & $28(1.3 \%)$ & $30(1.9 \%)$ & 0.13 \\
\hline COPD (incl. & $60(2.8 \%)$ & $61(3.9 \%)$ & 0.054 \\
\hline Emphysema/bronchitis) & $34(1.6 \%)$ & $58(3.7 \%)$ & $<0.001$ \\
\hline CKD & $113(5.2 \%)$ & $143(9.2 \%)$ & $<0.001$ \\
\hline Congestive Cardiac & $243(11.3 \%)$ & $212(13.6 \%)$ & 0.031 \\
\hline Failure & $603(28.0 \%)$ & $538(34.6 \%)$ & $<0.001$ \\
\hline Atrial Fibrillation & $71(3.3 \%)$ & $72(4.6 \%)$ & 0.037 \\
\hline Hypertension & $106(4.9 \%)$ & $173(11.1 \%)$ & $<0.001$ \\
\hline Hyperlipidemia & $174(8.1 \%)$ & $172(11.1 \%)$ & 0.002 \\
\hline Diabetes Mellitus & & & \\
\hline Cancers & & & \\
\hline SD - & & & \\
\hline
\end{tabular}

$\mathrm{SD}$ - standard deviation 
Table 2: Hazard Ratios (95\% CI) for dementia incidence post stroke after hospital discharge -anaemia

\begin{tabular}{|c|c|c|c|c|}
\hline Model & HR (95\% CI) for anaemia & $\begin{array}{c}\text { HR (95\% CI) for 20 g/dL unit } \\
\text { increase in HB }\end{array}$ & $\begin{array}{c}\text { HR (95\% CI) for anaemia* } \\
\text { HR (95\% CI) for 20 g/dL } \\
\text { unit increase in HB* }\end{array}$ \\
\hline & $\mathrm{N}=578$ failures / 7454 & $\mathrm{N}=578$ failures / 7454 & N=578 failures / 7454 & N=578 failures / 7454 \\
\hline A & $1.21(1.01,1.43)$ & $0.70(0.64,0.77)$ & $1.34(1.13,1.58)$ & $1.00(0.91,1.10)$ \\
\hline B & $1.21(1.01,1.44)$ & $0.88(0.80,0.97)$ & $1.03(0.86,1.22)$ & $1.01(0.92,1.11)$ \\
\hline C & $1.17(0.97,1.40)$ & $0.89(0.80,0.98)$ & $1.02(0.85,1.26)$ & $1.02(0.93,1.13)$ \\
\hline D & $1.13(0.94,1.36)$ & $0.91(0.82,1.01)$ & $1.00(0.89,1.22)$ & $1.04(0.94,1.15)$ \\
\hline E & $1.10(0.91,1.33)$ & $0.93(0.83,1.03)$ & $0.97(0.80,1.17)$ & $1.05(0.95,1.16)$ \\
\hline F & $1.10(0.92,1.33)$ & $0.95(0.85,1.06)$ & $0.97(0.80,1.17)$ & $1.05(0.95,1.16)$ \\
\hline
\end{tabular}

*Competing risk model with death as competing risk

A: age, sex adjusted

B: age, sex, stroke characteristics adjusted (subtype, OCSP)

C: age, sex, stroke type, OCSP and pre-stroke mRS adjusted

D: C + additional adjustments for key risk factors for vascular dementia AF, CHD, previous stroke/TIA, PVD, hypertension

E: D+ other co-morbidities

F: E + year of admission 
Figure 1: Kaplan Meier Curves showing the incidence of dementia in patients without anaemia (top line) and with anaemia (bottom line)

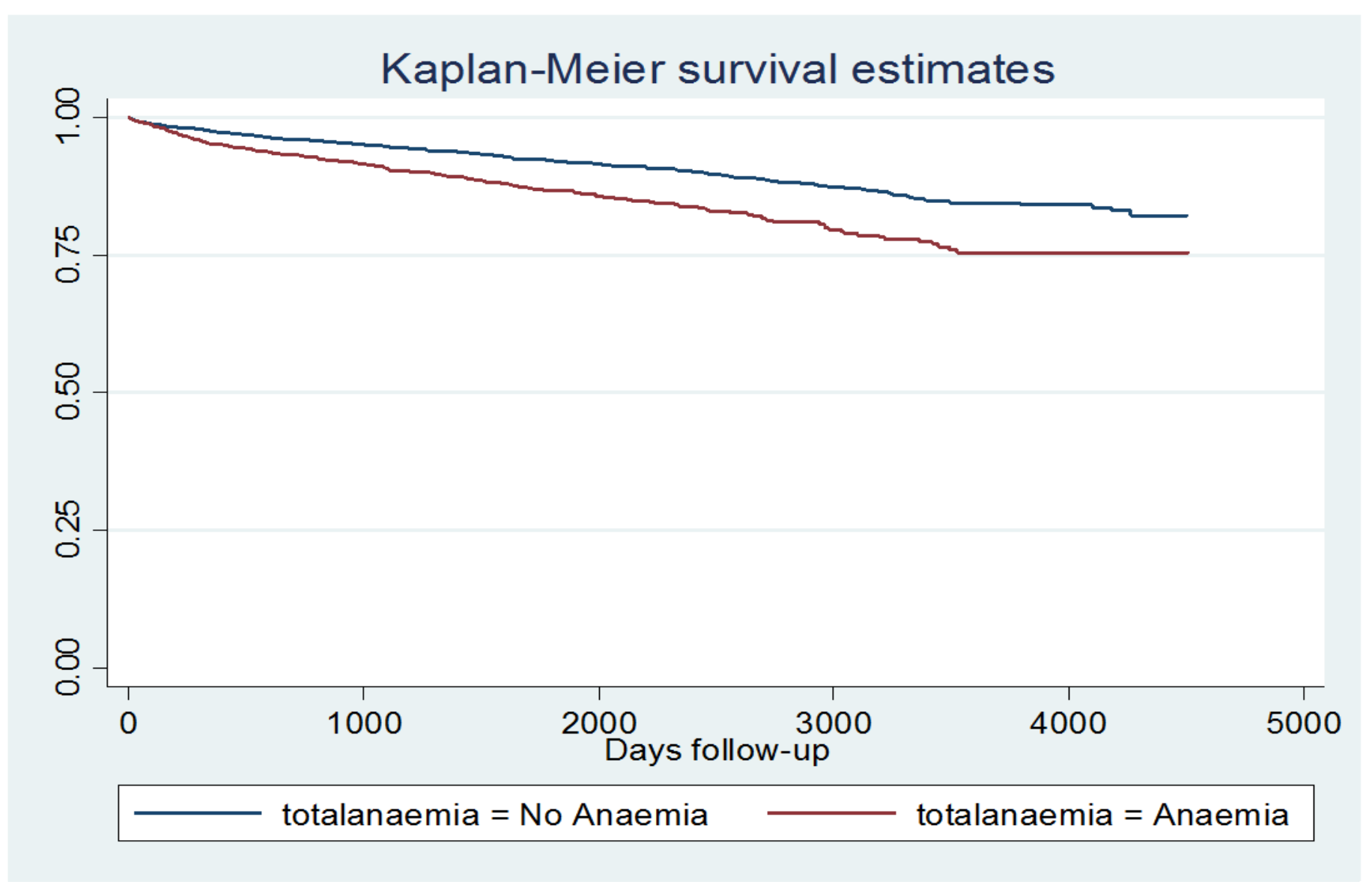

\begin{tabular}{|l|r|r|c|c|c|c|}
\hline & N at risk & & & & & \\
\hline No anaemia & 5019 & 2848 & 1413 & 755 & 216 & 0 \\
\hline Anaemia & 2435 & 1035 & 457 & 240 & 68 & 0 \\
\hline
\end{tabular}


Figure 2: Kaplan-Meier Curve for dementia incidence for individual year

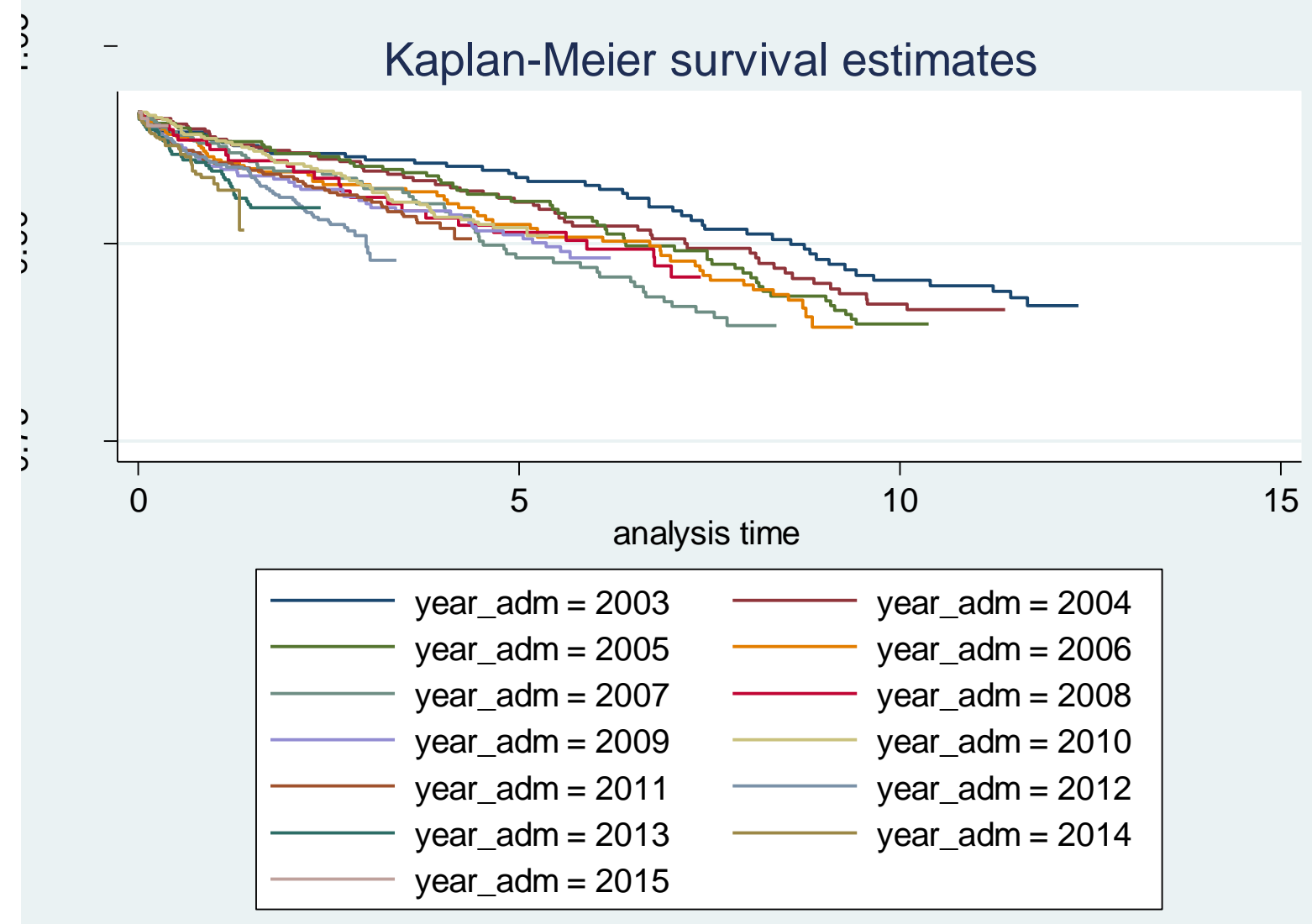

\begin{tabular}{|c|c|}
\hline Year (vs. 2003) & Hazard Ratios $(95 \%$ CI) \\
\hline $\mathbf{2 0 0 4}$ & $1.16(0.78,1.71)$ \\
\hline $\mathbf{2 0 0 5}$ & $1.27(0.86,1.86)$ \\
\hline $\mathbf{2 0 0 6}$ & $1.46(0.99,2.17)$ \\
\hline $\mathbf{2 0 0 7}$ & $1.75(1.18,2.59)$ \\
\hline $\mathbf{2 0 0 8}$ & $1.56(0.96,2.54)$ \\
\hline $\mathbf{2 0 0 9}$ & $1.76(1.15,2.69)$ \\
\hline $\mathbf{2 0 1 0}$ & $1.56(1.04,2.34)$ \\
\hline $\mathbf{2 0 1 1}$ & $1.99(1.32,2.99)$ \\
\hline $\mathbf{2 0 1 2}$ & $2.82(1.89,4.22)$ \\
\hline $\mathbf{2 0 1 3}$ & $2.99(1.88,4.76)$ \\
\hline $\mathbf{2 0 1 4}$ & $3.24(2.01,5.2)$ \\
\hline
\end{tabular}

Note 2015 data is not shown as it doesn't have full annul follow up. 
Supplementary Table 1: Annual incidence of dementia overtime in patients who survived to hospital discharge over 10 years follow up

\begin{tabular}{|c|c|c|c|c|c|c|c|c|c|c|c|c|c|}
\hline & & 2004 & 2005 & 2006 & 2007 & 2008 & 2009 & 2010 & 2011 & 2012 & 2013 & 2014 & 2015 \\
\hline \multirow[t]{2}{*}{2003} & $\mathrm{~N}$ at risk & 469 & 418 & 391 & 353 & 316 & 291 & 263 & 242 & 220 & 207 & 206 & 201 \\
\hline & Incidence $/ 1,000$ & 14.93 & 4.78 & 5.12 & 5.67 & 12.66 & 10.31 & 30.42 & 12.4 & 22.73 & 9.66 & 19.42 & 0 \\
\hline \multirow[t]{2}{*}{2004} & $\mathrm{~N}$ at risk & 538 & 469 & 424 & 385 & 350 & 307 & 281 & 252 & 232 & 218 & 214 & 211 \\
\hline & Incidence $/ 1,000$ & 9.29 & 19.19 & 9.43 & 20.78 & 2.86 & 19.54 & 24.91 & 7.94 & 21.55 & 22.94 & 9.35 & 0 \\
\hline \multirow[t]{2}{*}{2005} & $\mathrm{~N}$ at risk & & 528 & 452 & 408 & 377 & 343 & 311 & 288 & 263 & 250 & 245 & 237 \\
\hline & Incidence $/ 1,000$ & & 9.47 & 15.49 & 12.25 & 13.26 & 17.49 & 12.86 & 27.78 & 15.21 & 28 & 28.57 & 0 \\
\hline \multirow[t]{2}{*}{2006} & $\mathrm{~N}$ at risk & & & 493 & 427 & 361 & 328 & 304 & 275 & 254 & 239 & 240 & 232 \\
\hline & Incidence $/ 1,000$ & & & 16.23 & 30.44 & 8.31 & 9.15 & 23.03 & 10.91 & 23.62 & 4.18 & 33.33 & 12.93 \\
\hline \multirow[t]{2}{*}{2007} & $\mathrm{~N}$ at risk & & & & 490 & 414 & 362 & 326 & 297 & 254 & 232 & 228 & 221 \\
\hline & Incidence $/ 1,000$ & & & & 14.29 & 31.4 & 2.76 & 15.34 & 40.4 & 19.69 & 21.55 & 30.7 & 4.52 \\
\hline \multirow[t]{2}{*}{2008} & $\mathrm{~N}$ at risk & & & & & 265 & 227 & 194 & 174 & 160 & 146 & 145 & 142 \\
\hline & Incidence $/ 1,000$ & & & & & 18.87 & 22.03 & 25.77 & 22.99 & 6.25 & 6.85 & 20.69 & 14.08 \\
\hline \multirow[t]{2}{*}{2009} & $\mathrm{~N}$ at risk & & & & & & 460 & 398 & 361 & 324 & 309 & 308 & 301 \\
\hline & Incidence $/ 1,000$ & & & & & & 17.39 & 30.15 & 16.62 & 18.52 & 9.71 & 22.73 & 3.32 \\
\hline \multirow[t]{2}{*}{2010} & $\mathrm{~N}$ at risk & & & & & & & 692 & 611 & 543 & 508 & 497 & 482 \\
\hline & Incidence $/ 1,000$ & & & & & & & 11.56 & 18.00 & 18.42 & 21.65 & 20.12 & 6.22 \\
\hline \multirow[t]{2}{*}{2011} & $\mathrm{~N}$ at risk & & & & & & & & 651 & 575 & 540 & 531 & 521 \\
\hline & Incidence $/ 1,000$ & & & & & & & & 24.58 & 20.87 & 18.52 & 16.95 & 11.52 \\
\hline \multirow[t]{2}{*}{2012} & $\mathrm{~N}$ at risk & & & & & & & & & 671 & 587 & 565 & 547 \\
\hline & Incidence $/ 1,000$ & & & & & & & & & 23.85 & 34.07 & 28.32 & 16.45 \\
\hline \multirow[t]{2}{*}{2013} & $\mathrm{~N}$ at risk & & & & & & & & & & 487 & 426 & 395 \\
\hline & Incidence $/ 1,000$ & & & & & & & & & & 26.69 & 44.6 & 5.06 \\
\hline \multirow[t]{2}{*}{2014} & $\mathrm{~N}$ at risk & & & & & & & & & & & 671 & 612 \\
\hline & Incidence $/ 1,000$ & & & & & & & & & & & 37.26 & 13.07 \\
\hline \multirow[t]{2}{*}{2015} & $\mathrm{~N}$ at risk & & & & & & & & & & & & 191 \\
\hline & Incidence $/ 1,000$ & & & & & & & & & & & & 10.47 \\
\hline
\end{tabular}

$\mathrm{N}$ at risk $=$ total number of patients - Number of deaths occurring within the year 